\title{
Rheological properties of PDMS/clay nanocomposites and their sensitivity to microstructure
}

\author{
Y. Zhu, R. Cardinaels, J. Mewis, P. Moldenaers \\ Lab for Applied Rheology and Polymer Processing \\ Department of Chemical Engineering \\ KU Leuven \\ Willem de Croylaan 46, Box 2423, B-3001 Leuven, Belgium \\ Jan.Mewis@cit.kuleuven.be
}

Final accepted draft

Cite as: Y. Zhu, R. Cardinaels, J. Mewis, P. Moldenaers, Rheol. Acta, 48(9), pp. 1049-1058 (2009)

The original publication is available at www.springerlink.com.

http://link.springer.com/article/10.1007\%2Fs00397-009-0387-3 


\title{
Rheological properties of PDMS/clay nanocomposites and their sensitivity to microstructure
}

\author{
YUTIAN ZHU, RUTH CARDINAELS, JAN MEWIS, PAULA MOLDENAERS
}

\begin{abstract}
The effect of microstructure on the rheology of clay/polymer nanocomposites is investigated using dispersions of organically treated clay in nearly Newtonian poly(dimethylsiloxane). Degree of dispersion and floc size are altered by using two different dispersion procedures and by changing the shear history. The scaling for dynamic moduli of attractive colloids applies, except for a possible relaxation mechanism at very low frequencies. The time to reach the crossover at a given frequency is found to be extremely sensitive to the dispersion procedure used. Hydrodynamic and elastic components of the steady state stress on the other hand, evolve in a very similar fashion for the different systems. Although the relaxation times of the elastic stress components change drastically with flow-induced changes in structure, the dispersion process has hardly an effect at all. Intermittent start-up flows in the forward and reverse direction show that anisotropy persists long after the flow has been arrested, even at shear rates where no large reversible flocs are present. The degree of dispersion had only a limited effect on the anisotropy. Finally, the effect of shear on structure recovery has been studied. Very low shear rates are found to increase the rate of recovery, even for small strains.
\end{abstract}

Key words: polymer/clay nanocomposites - dispersion procedure - scaling for dynamic moduli - stress jumps - flow reversals - transient viscosity

\section{Y. Zhu ${ }^{l}$, R. Cardinaels, J. Mewis( ), P. Moldenaers}

Department of Chemical Engineering, Katholieke Universiteit Leuven, W. de Croylaan 46, B-3001 Leuven (Heverlee), Belgium

${ }^{1}$ Present address: Department of Chemistry, The University of Memphis, 425 Smith Hall, Memphis, TN 38152, USA

(J) Email: Jan.Mewis@ @it.kuleuven.be

Fax: 03216322991

Phone : 03216322361 


\section{Introduction}

The remarkable improvement in material properties of clay/polymer nanocomposites over those of the virgin polymer is a result of the shape and size of the clay particles. Fully exfoliated clay sheets have a layer thickness of about $1 \mathrm{~nm}$ and an aspect ratio of up to 1000. A very small volume fraction of such particles can drastically increase stiffness and strength (e.g. Wang et al. 1998; Liu et al. 1999), as well as dimensional stability (Gilman 1999; Zeng and Lee 2001). Addition of clay can also improve flame retardancy (Vaia et al. 1999; Gilman et al. 2000) and decrease gas permeability (e.g. Messersmith and Giannelis 1995; Yano et al. 1997). Polymer-layered clay nanocomposites can be prepared by either in-situ polymerisation, intercalation from a polymer solution or direct intercalation by molten polymer. Most often the final properties improve with increasing degree of dispersion. Especially with clays there might be limitations to this rule (see e.g. Bousmina 2006).

The rheological properties of liquid nanocomposites are obviously important when processing such materials. They depend on size, shape and volume fraction of the particles as well as on the interparticle forces. In addition, the rheology of polymerparticle hybrids is very sensitive to the particulate microstructure. It makes rheometry one of the most sensitive methods to probe this microstructure. The latter not only controls the flow behavior but is in turn affected by the flow, resulting in a complex interplay between microstructure and rheology.

Adding clay particles to a polymer melt changes its rheological properties in various ways. In the high frequency range the moduli gradually increase with increasing clay volume fraction (e.g. Vermant et al. 2007). This is the typical behaviour of all filled polymers, whether the particles are colloidal or not (Carreau et al. 1996; Aral and Kalyon 1997; Le Meins et al. 2002). Small colloidal particles in general, and clays in particular, cause a much stronger increase in moduli and viscosities at low frequencies and low shear rates. Already at low particle concentrations a low frequency plateau develops for the storage moduli and a yield stress appears in the viscosity curve. These features signal a transition to solid-like behaviour (Krishnamoorti and Yurekli 2001, Solomon et al. 2001), which suggests that a space-filling particulate network has developed. The particle concentration at which this transition occurs can be expected to be very sensitive to the degree of dispersion (Zhao et al. 2005) and to the interparticle forces. The mechanisms involved in this network formation and their modeling have not been fully resolved. In particular the role of Brownian motion and interparticle attraction forces is still debated (Solomon et al. 2001; Ren et al. 2003; Mobuchon et al. 2007; Nazockdast et al. 2008). 
Among the other rheological features of clay/polymer nanocomposites, structural anisotropy and thixotropy are prominent ones. As in clay dispersions in low viscosity media (Pignon et al. 1998), the dispersions in polymer melts display flow-induced anisotropy. It clearly shows up in flow reversal experiments. The anisotropy is characterized by pronounced shear history effects, it only decays slowly after cessation of flow (Solomon et al. 2001; Letwimolnun et al. 2007; Vermant et al. 2007). The time effects do not only include normal viscoelasticity but also thixotropy (or aging and shearinduced rejuvenation), i.e. a gradual decrease of the viscosity during shear that slowly recovers after cessation of flow or after reducing the shear rate (Barnes 1997, Mewis and Wagner 2009). Hence, the various protocols that are used to analyze thixotropy can also be applied to clay/polymer nanocomposites. As in some other systems, the shear effects often cannot be recovered fully at rest but are reversible during flow (Mobuchon et al. 2007).

The objective of the present work is to evaluate the sensitivity to structural differences of the various rheological features, including transient and steady state data. Results will be compared for samples based on the same components but prepared in a different manner so as to alter the degree of dispersion. Further changes in microstructure have been generated by applying different shear histories.

\section{Materials and methods}

The matrix phase of the composites consists of a poly(dimethylsiloxane) (PDMS Rhodorsil 47V100000 from Rhodia). At $23{ }^{\circ} \mathrm{C}$ it has a density of $971 \mathrm{~kg} / \mathrm{m}^{3}$ and a viscosity of 102 Pa.s. A relatively low molecular weight PDMS has been selected to keep the relaxation time of the polymer phase small enough $(<30 \mathrm{~ms})$ to enable an accurate determination of the hydrodynamic contribution to the steady state viscosities. The clay is a refined white hectorite (EA-3131, provided by Elementis Specialties). It has been made compatible with low polarity media by treating it with a dimethyldihydrogenated tallow quart. The spacing in the layered structure of the clay was determined with $\mathrm{x}$-ray diffraction. The measured value was $3.7 \mathrm{~nm}$, this large interlayer distance confirms the presence of the quart.

The PDMS/clay nanocomposites were prepared with a solution intercalation method. Chloroform was used as the solvent as it is compatible with the clay and with the PDMS. An ultrasound treatment (Ikasonic U 400 s Control, Germany) was used to improve the 
dispersion degree of the clay in solution. Two degrees of dispersion were obtained by employing two different preparation procedures. In the first one, labeled P1, clay and PDMS were blended together in chloroform, the mixture was then stirred and subjected to ultrasound for 10 hours. In the second process (P2), only the clay was dissolved in chloroform. The clay/solvent mixture was also dispersed with ultrasound for 10 hours. Subsequently, this dispersion was mixed with polymer dissolved in chloroform, and then stirred further overnight. As only a difference in structure was aimed at, no attempt was made to optimize the dispersion process. From the samples dispersed according to either treatment P1 or P2, the solvent was evaporated in a rotary device (Rotovapor BÜCHI) at $60 \mathrm{rpm}$ and $45^{\circ} \mathrm{C}$. After the evaporation stage, the PDMS/clay samples were dried further for $24 \mathrm{~h}$ using a vacuum drier to eliminate the chloroform. With the P1 procedure a sample of $4.0 \mathrm{wt} \%$ clay has been prepared, samples with $2.5,3,3.2$ and $4.0 \mathrm{wt} \%$ clay were prepared according to procedure P2. SAXS data for the pure clay and samples with 4 wt\% clay, prepared according to method P1 and P2, are shown in Fig. 1. The presence of diffraction peaks for the polymer nanocomposites indicates that both treatments do not result in full exfoliation, with clay tactoids remaining in both samples. The SAXS data suggest a slightly higher degree of exfoliation when using the P2 procedure. However, other factors such as mixed layer spacings can also cause a reduction in peak height (Morgan and Gilman 2003). Therefore, essentially rheology has been used to discriminate between the samples. 


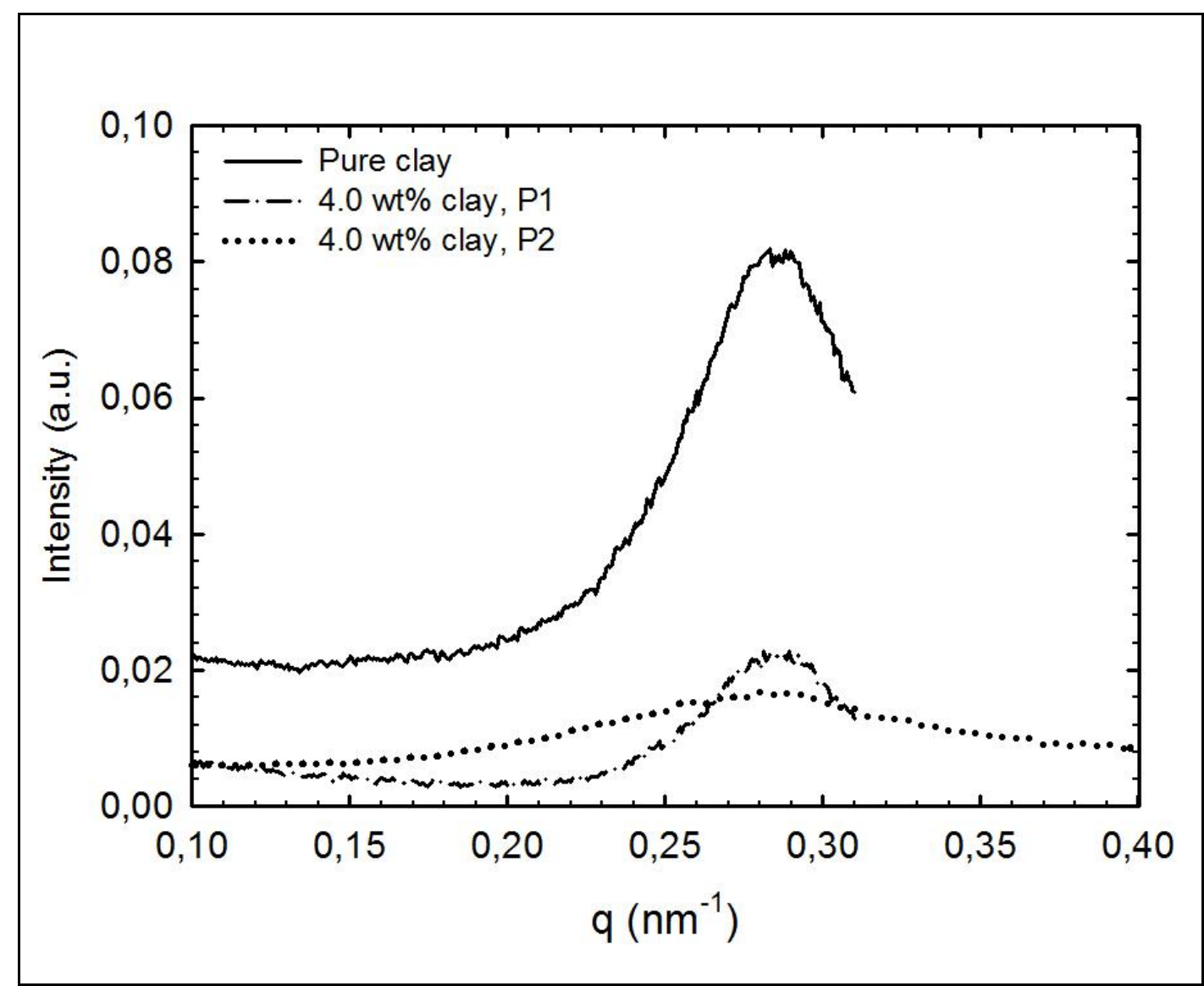

Fig. 1 SAXS patterns of pure clay and of two polymer/clay nanocomposites obtained with a different dispersion procedure

The rheological measurements were either performed on a strain-controlled rheometer (ARES-TA Instruments) or a stress-controlled rheometer (Physica MCR 501-Anton Paar) at $23{ }^{\circ} \mathrm{C}$. A cone and plate geometry $(\mathrm{R}=12.5 \mathrm{~mm}$, cone angle $=0.04 \mathrm{rad})$ was used in all experiments. It ensured a constant shear history throughout the sample, which is required for transient measurements on thixotropic samples. In the dynamic measurements the peak strain was kept at 0.01 , within the linearity limit. To monitor structure recovery during shear, the steady flow had to be interrupted intermittently. These measurements were performed on the Physica MCR 501.

\section{Results and Discussion}

\section{Dynamic moduli}

The dynamic moduli of clay/polymer nanocomposites depend on the shear history. To compare the effect of the preparation procedure on the moduli, samples with 4 wt\% clay and prepared according to procedures P1 and P2 were sheared for 10 minutes at $5 \mathrm{~s}^{-1}$. Subsequently, the modulus-frequency curves were measured after rest times $\left(\mathrm{t}_{\mathrm{r}}\right)$ between 
two and sixteen hours (see Figs. 2a and $2 \mathrm{~b}$ ). The high frequency storage and loss moduli hardly change with time for both samples. Also the difference between the preparation procedures remains marginal. At low frequencies both samples show a plateau in the G'$\omega$ curves. It indicates that both samples contain a percolating network of clay particles. The loss moduli also level off at low frequencies, some G"-curves show a tendency to increase again at even lower frequencies. This pattern is common for gelled dispersions. The change in preparation procedure causes a difference of a factor of 4 in plateau storage moduli. This substantial difference confirms the sensitivity of rheological measurements to changes in microstructure. The slow increase of the low frequency moduli with time, which still continues after long times, is a characteristic ageing process seen in many gelled and glassy dispersions, including clay/polymer nanocomposites (e.g. Mobuchon et al. 2007). The evolution can be described over large time spans by a power law relation and is attributed to local rearrangements in the non-equilibrium structure induced by e.g. thermal motion ("thermal hopping") or internal stresses (Kobelev and Schweizer 2005).

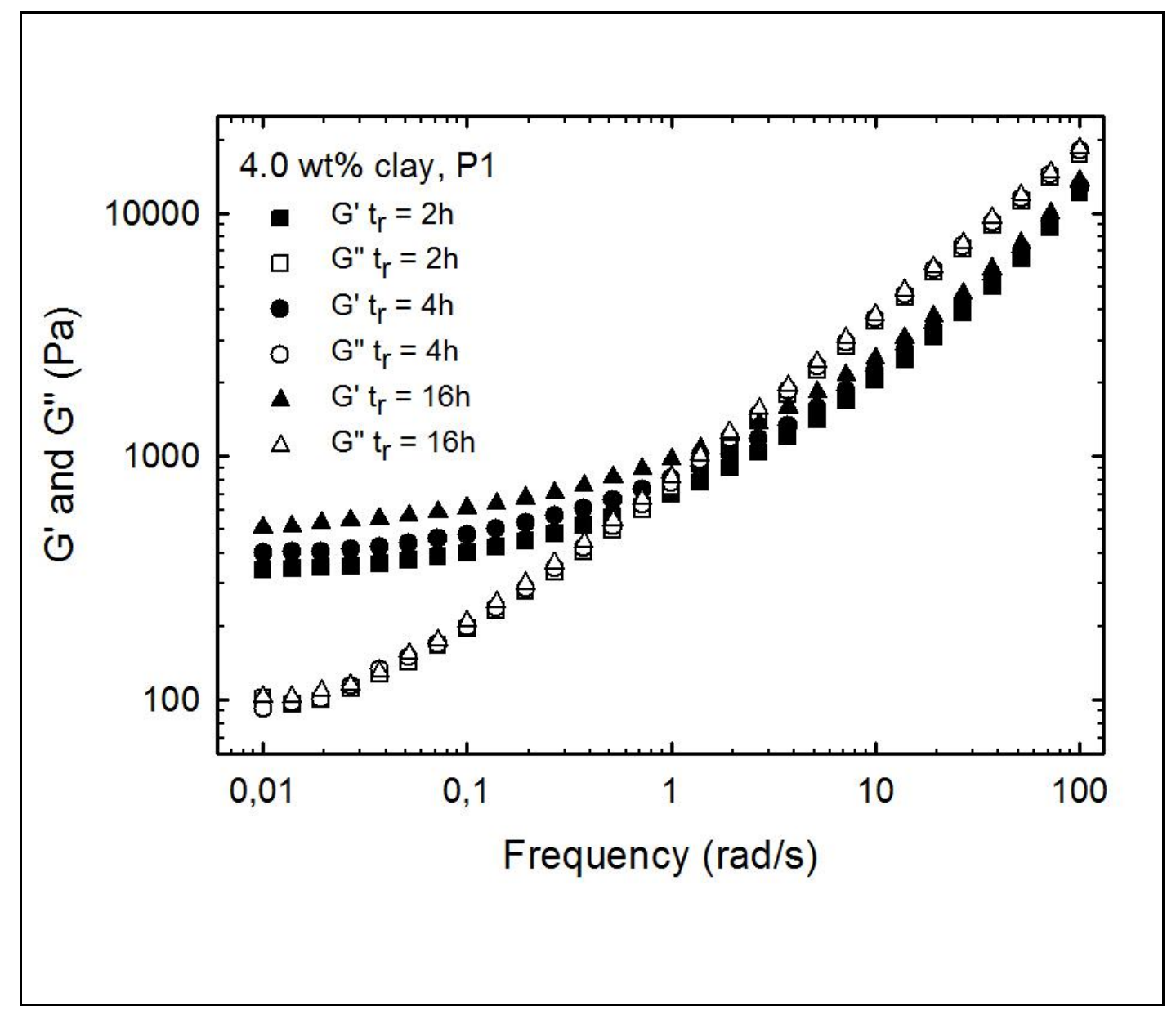




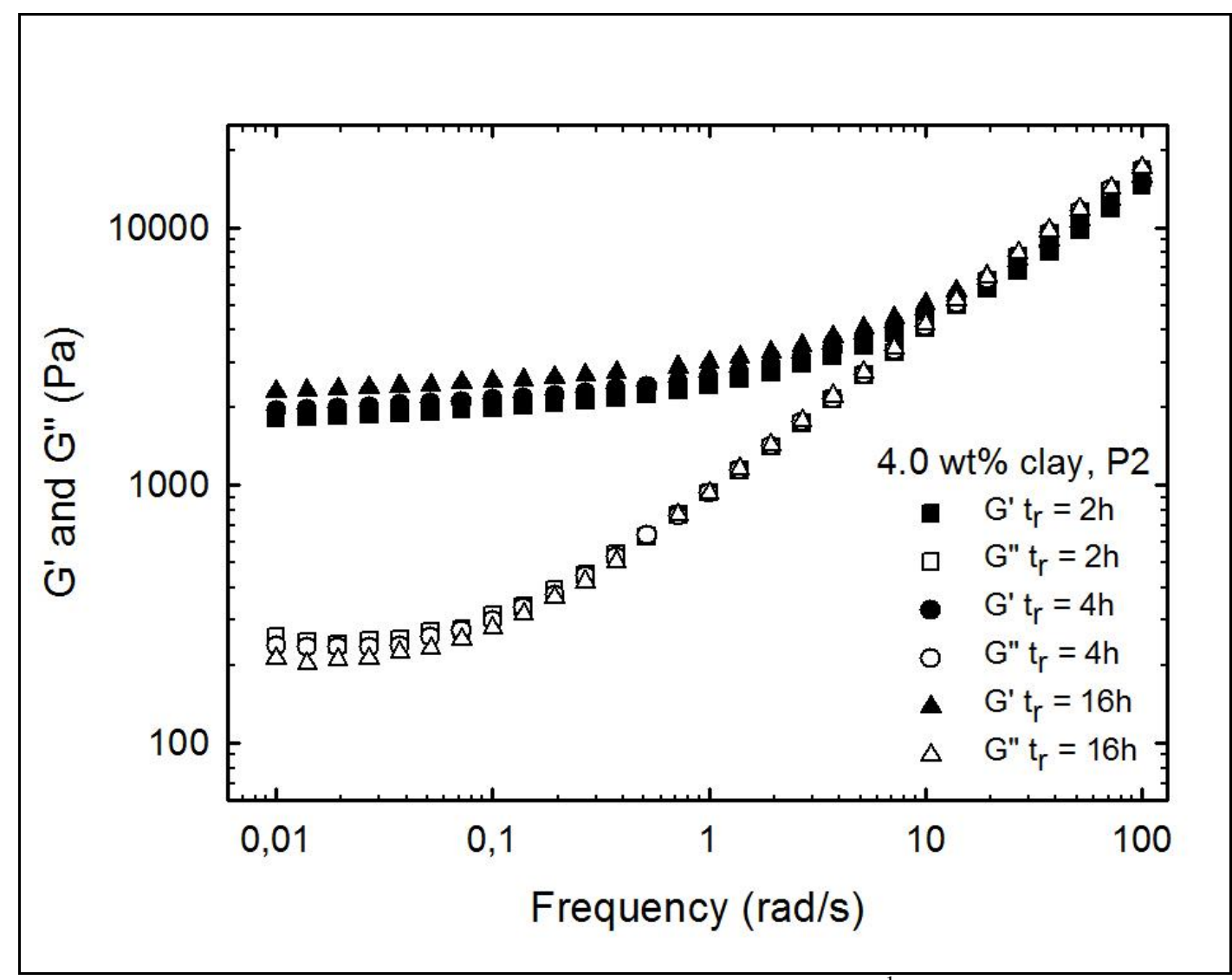

Fig. 2 Recovery of the dynamic moduli after shearing at $5 \mathrm{~s}^{-1}$ for 10 minutes, strain amplitude $=0.01$; (a) $4.0 \mathrm{wt} \%$ clay, preparation procedure $\mathrm{P} 1$; (b) $4.0 \mathrm{wt} \%$ clay, preparation procedure $\mathrm{P} 2$

D. Weitz and coworkers (Trappe and Weitz 2000; Prasad et al. 2003) have reported a universal scaling for the dynamic moduli of attractive colloidal systems in Newtonian media. Data for samples with different volume fractions or strengths of the interparticle interaction could be superposed onto a single master curve, where the parameters of the crossover point for the curves of G' and $G^{\prime \prime}\left(G_{c}\right.$ and $\left.\omega_{c}\right)$ could be used as scaling factors. This scaling has been used to superimpose the modulus-frequency curves for a clay/polymer composite after different rest periods by Sung et al. (2008). The same scaling has been applied here on the modulus-frequency curves of Fig. 2 (see Fig. 3). Also data for a $2.5 \mathrm{wt} \%$ dispersion, prepared according to procedure P2, are included in Fig. 3. The various modulus-frequency curves superimpose quite well, with the exception of the loss moduli at low frequencies. The latter data seem to indicate an upturn in the G" curves, that could be caused by a relaxation mode at very low frequencies. In that case this mode does not follow the scaling. Such a deviating low frequency relaxation has also been observed in $\mathrm{TiO}_{2} /$ polymer nanocomposites (Romeo et al. 2008). The low frequency relaxation seems to reflect the dynamics of the weakest links between the large aggregates that form the network. Strengthening of these links would slow down the 
network dynamics without substantially changing the stiffness of the network. The phenomenon should then be associated with the heterogeneity of the network structure.

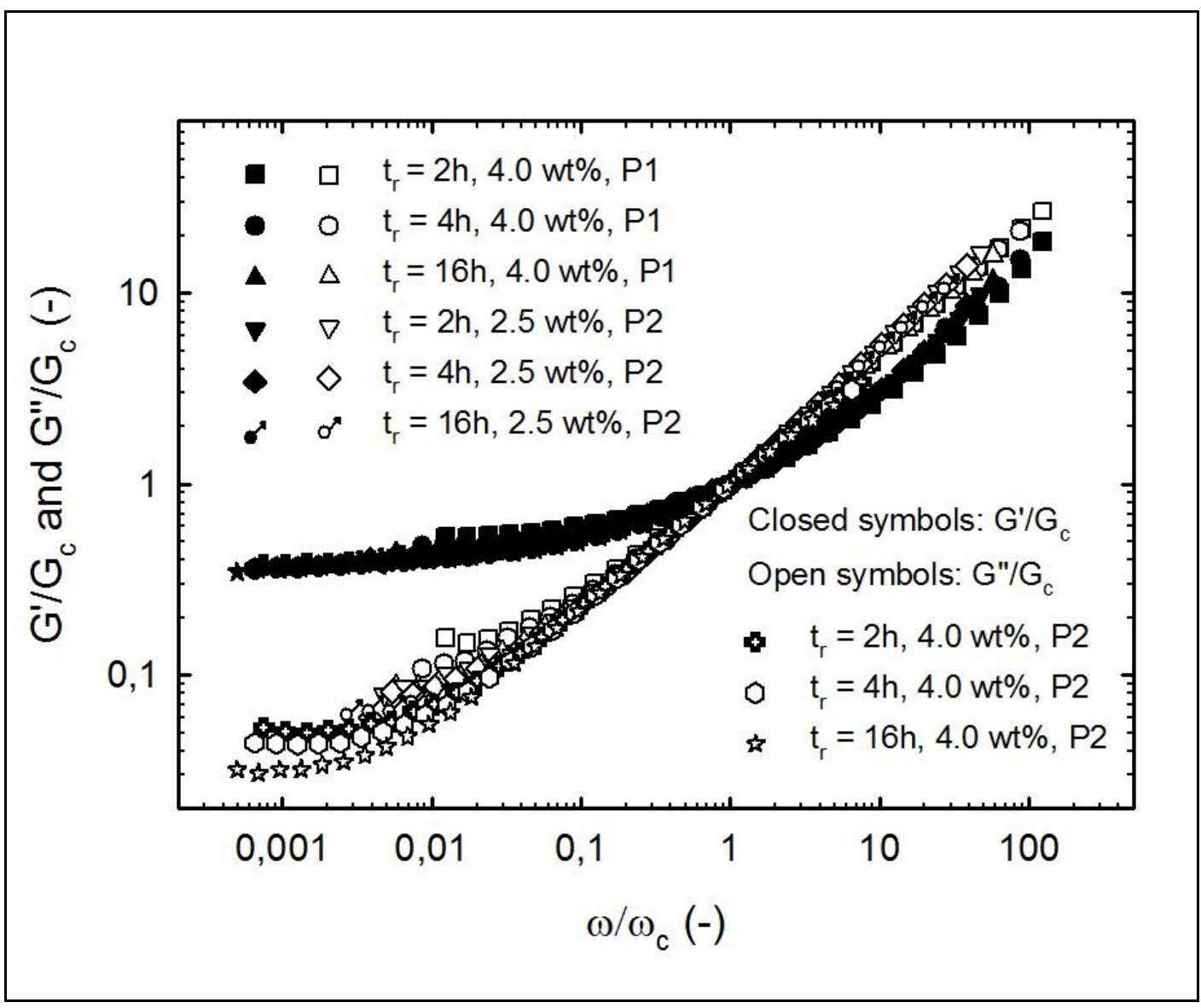

Fig. 3 Scaled modulus-frequency curves after various rest times (data of Fig. 2 and of a $2.5 \mathrm{wt} \%$ sample prepared according to procedure $\mathrm{P} 2$ )

In Fig. 4 the values of $G_{c}$ are plotted as a function of $\omega_{c}$ for the 2.5 and $4 \mathrm{wt} \%$ samples of Fig. 3 as well as for a sample with $3.2 \mathrm{wt} \%$ of clay, prepared according to procedure $\mathrm{P} 2$. The different clay loadings result in parallel lines in a logarithmic plot. The crossover parameters for the two $4 \mathrm{wt} \%$ samples are quite different but they are on the same line. As in earlier work (Sung et al. 2008), the $\mathrm{G}_{\mathrm{c}}-\omega_{\mathrm{c}}$ relation is not a linear one as predicted by the scaling. It can be seen in Fig. 2 that the crossover point occurs in the straight section of the logarithmic G' $(\omega)$ curves. This part of the curves is nearly parallel for the various samples with a slope that is slightly smaller than unity and it nearly coincides for the two 4 wt\% samples. The lines in Fig. 4 reflect the relative positions and slopes of the high frequency $G$ "- $\omega$ curves for the different samples. In the high frequency range, the contributions from the colloidal forces should become negligible. The hydrodynamic contributions of the particles, based on their "effective" volume, should dominate the material response under these conditions. This contribution seems not to be strongly 
affected by the preparation procedure at a given particle concentration. The medium viscosity is the same for all samples and does not provide a shift factor for $\omega_{\mathrm{c}}$ to superimpose the lines in Fig. 4 as it does for simple aggregating samples (Prasad et al. 2003). The high frequency viscosities do not lead to superposition either.

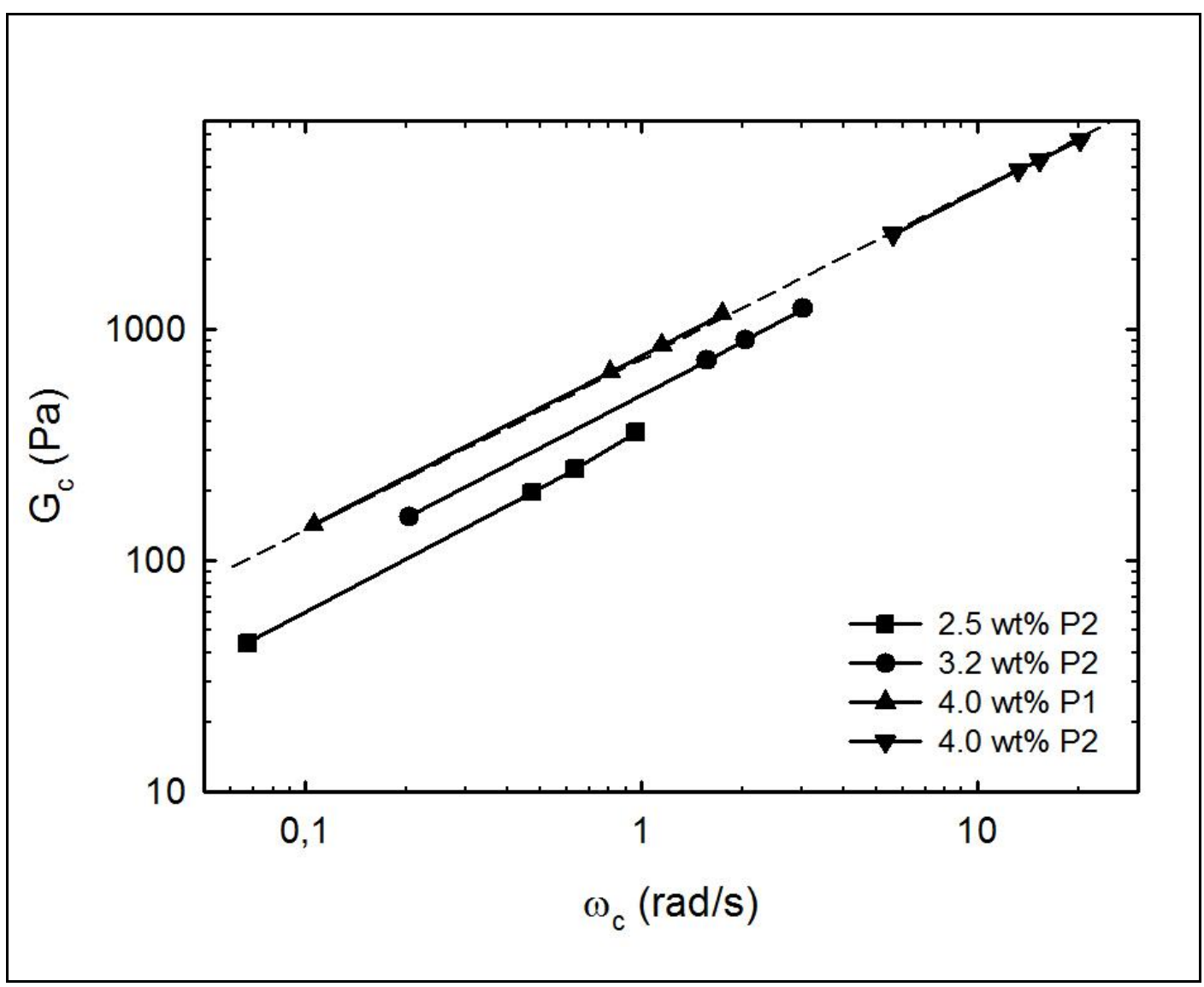

Fig. 4 Crossover moduli $G_{c}$ as a function of crossover frequency $\omega_{c}$ for suspensions with different clay contents and varying waiting times

The initial recovery of the structure after cessation of flow has also been investigated. The shear history is identical as that used in Fig. 2, i.e. shearing for 10 minutes at $5 \mathrm{~s}^{-1}$. For the samples of Fig. 3, the changes during the first hour have been probed by monitoring the evolution of the linear dynamic moduli at $1 \mathrm{rad} / \mathrm{s}$. The results are shown in Fig. 5. For all samples the growth in storage moduli is more pronounced than that in the loss moduli, as is common for structured materials. 


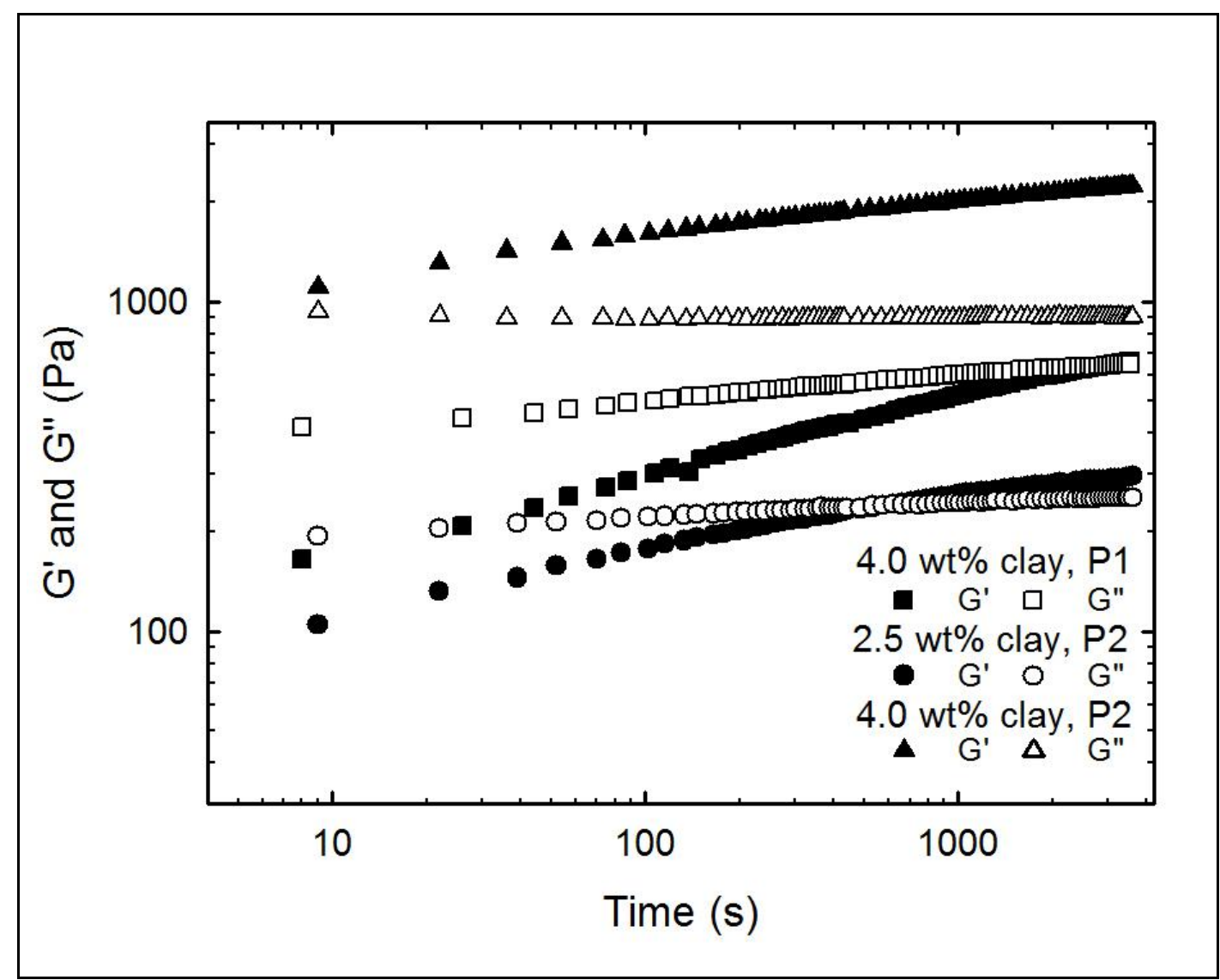

Fig. 5 Evolution of the dynamic moduli at $1 \mathrm{rad} / \mathrm{s}$ after shearing for 10 minutes at $5 \mathrm{~s}^{-1}$, effect of particle concentration and preparation procedure

Notwithstanding the quite high viscosity of the suspending medium, the initial rate of change for the storage moduli is high. The data in Fig. 5 can be used to estimate the time necessary to reach the crossover point at a frequency of $1 \mathrm{rad} / \mathrm{s}$. When prepared with the P2 procedure, the crossover for the $4 \mathrm{wt} \%$ sample occurs before the first data point, by extrapolation it is estimated to happen after approximately $3 \mathrm{~s}$. For the sample with the same clay content, but prepared according to procedure P1, it takes $3000 \mathrm{~s}$ to reach the crossover point. Consequently, the time to reach the crossover point provides a sensitive test to discriminate between slightly different dispersion procedures. The concentration effect is also pronounced, the corresponding time for the $2.5 \mathrm{wt} \% \mathrm{P} 2$ sample is $500 \mathrm{~s}$. Modifications in microstructure induced by flow also result in drastic changes of the time to crossover. Structure recovery for the $4 \mathrm{wt} \% \mathrm{P} 1$ sample after shearing at 1,2 or $5 \mathrm{~s}^{-1}$ gives crossover times of respectively 40, 1100 and 3000 s. Hence, both the degree of dispersion and the floc size affect the results. The clay/polymer hybrids used here do not produce "critical gels" (Winter and Chambon 1986) and do not reach the crossover point at the same time for all frequencies. Therefore the measured time differences depend on the frequency used. 


\section{Stress jumps}

Stresses in flowing suspensions, as in other complex fluids can in principle be separated into a viscous or hydrodynamic contribution and an elastic contribution related to flowinduced changes in microstructure (Batchelor 1970). Hydrodynamic stress components react instantaneously to sudden changes in shear rate. The elastic components, on the other hand, result in a stress transient because their changes require structure rearrangements. Hence, measuring the stress jump after arresting the flow provides a means to distinguish between these stress contributions, provided the necessary precautions are taken to eliminate instrumental artifacts (Mackay et al. 1992; Dullaert and Mewis 2005). For the ARES device used here, this consists in eliminating the electronic filter, dropping the shear rate to $0.0001 \mathrm{~s}^{-1}$ rather than to zero, and using a sufficiently fast data acquisition system. The stress jump technique has been used before on various materials, including colloidal suspensions (Mackay and Kaffashi 1995; Dullaert and Mewis 2005) and clay/polymer composites (Mobuchon et al. 2007). To avoid interference from the polymer matrix, a PDMS has been selected with a mean relaxation time not longer than the time constant of the device. Reliable data could be taken starting $30 \mathrm{~ms}$ after changing the shear rate.

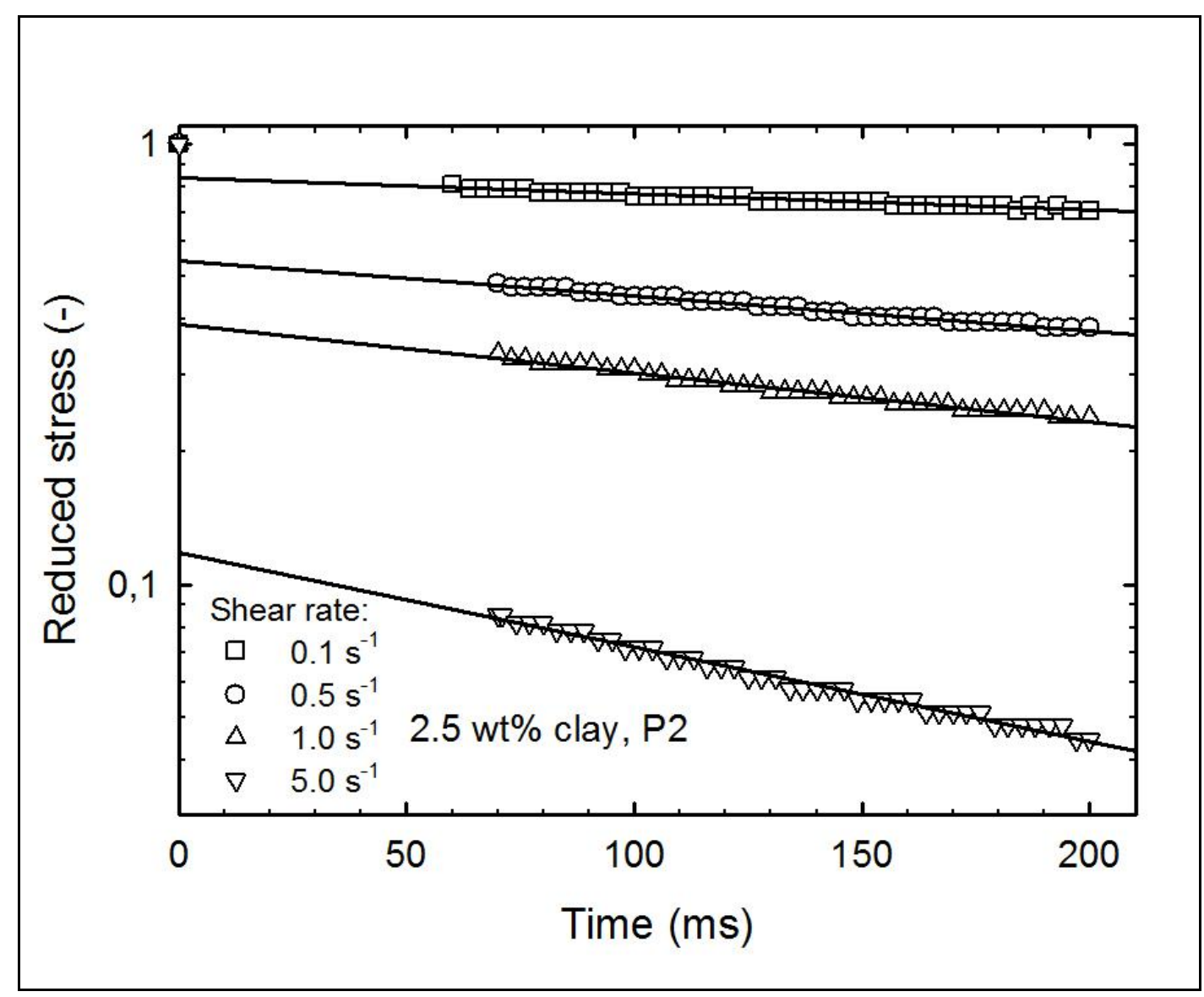


Fig. 6 Stress jump measurements: initial stress relaxation after shearing at $0.1,0.5,1.0$ and $5.0 \mathrm{~s}^{-1}$ for the sample with $2.5 \mathrm{wt} \%$ clay (preparation procedure $\mathrm{P} 2$ )

Fig. 6 illustrates the stress jump measurements on the sample with $2.5 \mathrm{wt} \%$ clay (P2). The stresses are scaled with the steady state value under shear. The initial relaxation after the stress jump is exponential and therefore a semilogarithmic plot is used. A linear extrapolation of the stress relaxation line to time zero then provides the elastic stress contribution. Over the shear rate range covered here the steady state stress evolves from mainly elastic, at low shear rates, to dominantly hydrodynamic at high shear rates. The slopes of the relaxation curves indicate that the initial relaxation times decrease with increasing shear rate, although not drastically. This decrease is most likely the result of smaller structural entities being involved.

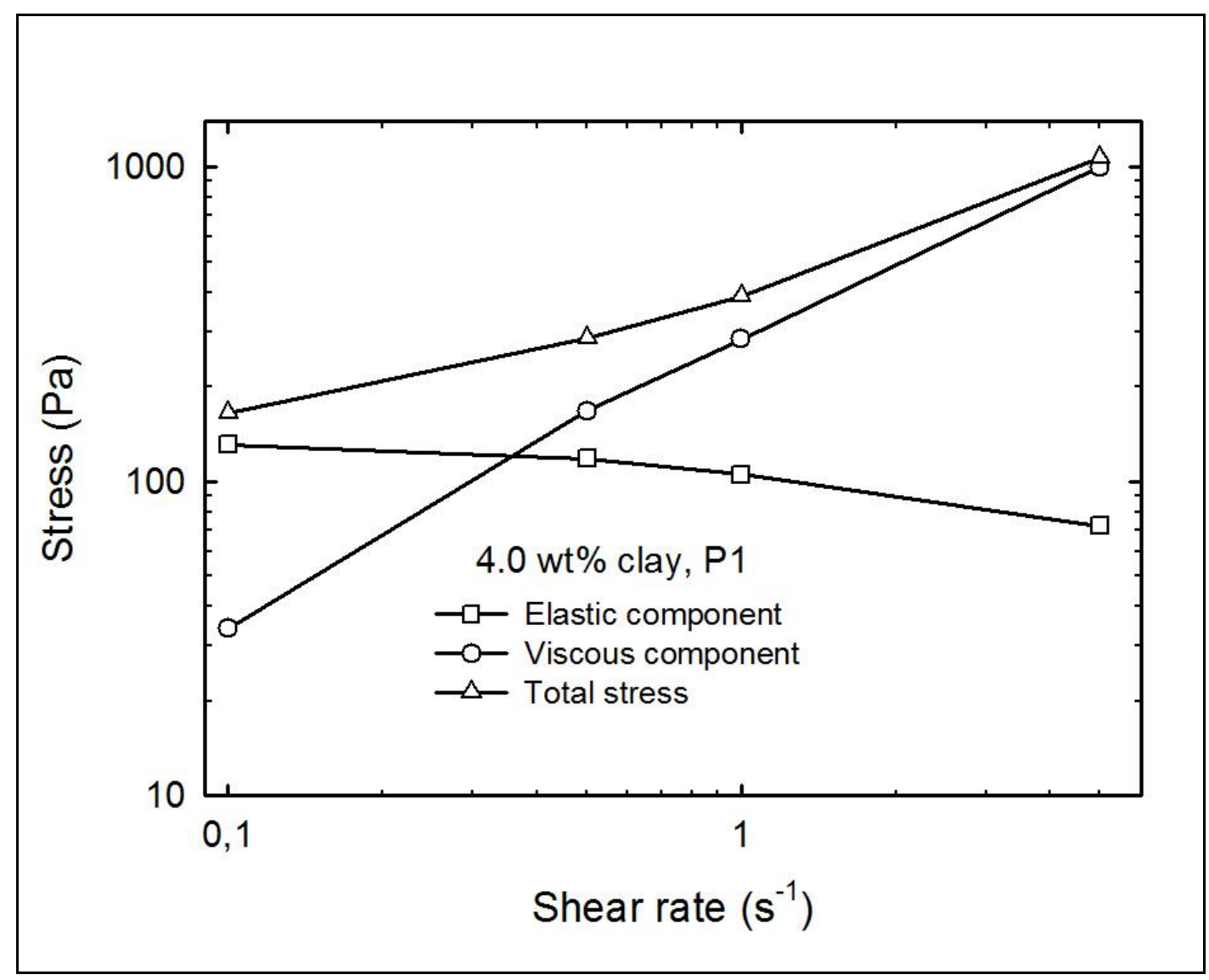




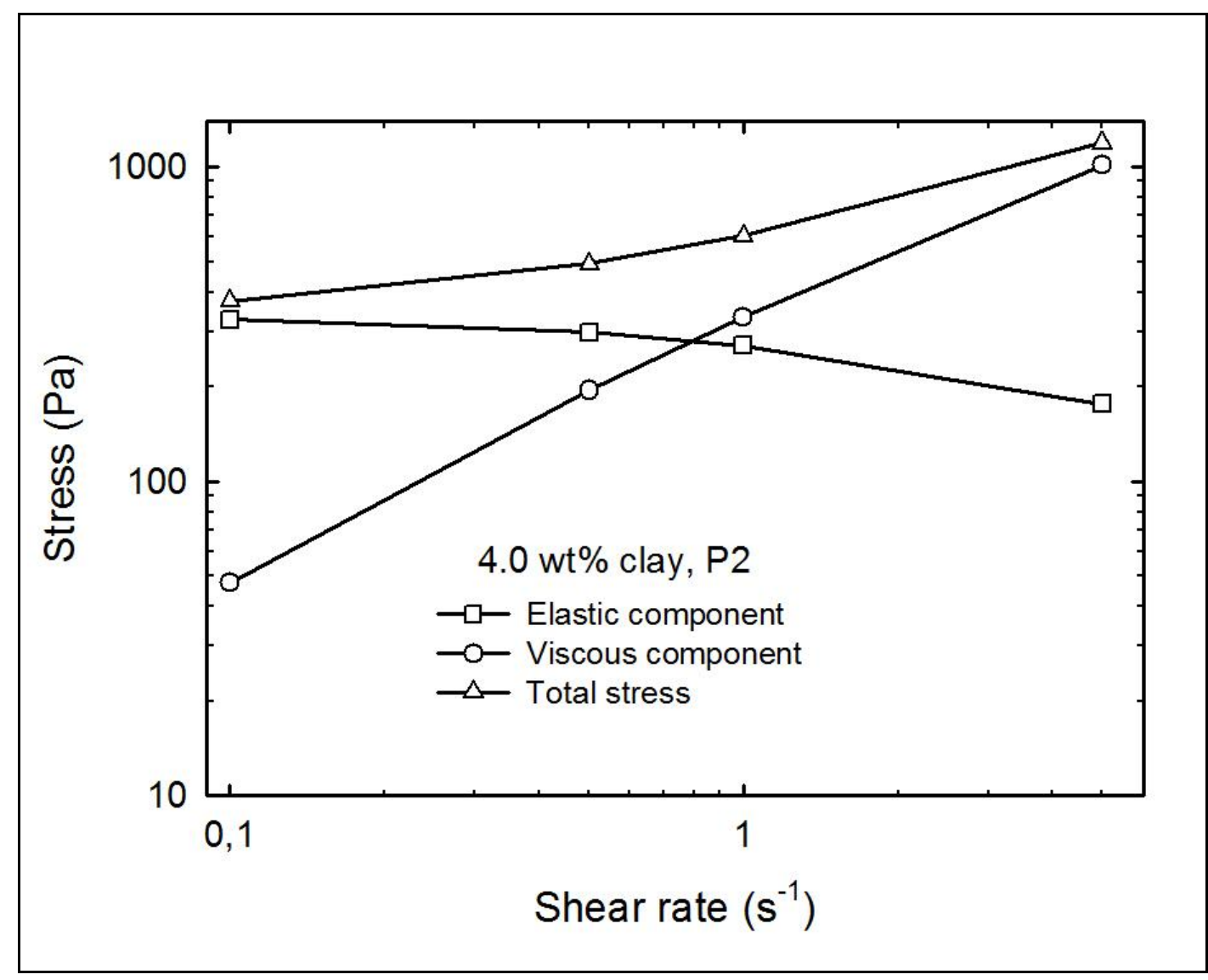

Fig. 7 Effect of preparation procedure on the elastic ( $\square$ ) and hydrodynamic (o) contributions to the total steady state stress $(\Delta)$; (a) $4.0 \mathrm{wt} \%$ clay, preparation procedure $\mathrm{P} 1$; (b) $4.0 \mathrm{wt} \%$ clay, preparation procedure $\mathrm{P} 2$

The steady state stresses and their viscous and elastic components are shown in Fig. 7 for the $4 \mathrm{wt} \%$ samples of Fig 2. The curves for the two systems look very similar in shape. Extrapolation of the data to lower shear rates suggests that the total stress approaches the dynamic yield stress, the latter is essentially an elastic stress. The hydrodynamic contribution is only slightly shear thinning, even tending towards a constant viscosity at low shear rates. The strongly non-Newtonian overall response is caused by the elastic stresses. These actually decrease with increasing shear rate because of loss of structure, a common effect for flocculated suspensions (Dullaert and Mewis 2005). The difference in preparation procedure between the two $4 \mathrm{wt} \%$ samples cannot be detected in the high shear hydrodynamic response. This response could be understood to be an emanation of an "effective hydrodynamic diameter", which then remains unaffected by the change in sample preparation. The small difference in total stress at $5 \mathrm{~s}^{-1}$ is caused by the difference in the small but non-negligible elastic stresses at such a high shear rate. The more developed microstructure resulting from preparation procedure P2 compared with P1 is most visible at low shear rates. In Fig. 7 it can be seen that larger flocs cause in particular larger elastic stresses. Still, the effect of the preparation procedure on the elastic stresses 
is somewhat smaller than that on the plateau storage moduli. The ratio between the hydrodynamic contributions of the two $4 \mathrm{wt} \%$ samples at $0.1 \mathrm{~s}^{-1}$ is similar to that of the loss moduli at low frequencies.

A final characteristic that can be deduced from the stress jump measurements is the relaxation time of the initial exponential relaxation. It can be deduced from the initial slopes of the stress relaxation curves in a semilogarithmic plot (Fig. 6). The results for the two $4 \mathrm{wt} \%$ samples and the $2.5 \mathrm{wt} \%$ sample of Fig. 6 are displayed in Fig. 8. The pronounced similarity between the three samples could suggest that the microstructure does not play a significant role here and that the medium viscosity governs the relaxation. This is not consistent with the strong dependence of the relaxation time on shear rate, i.e. nearly inversely proportional to the square root of the shear rate. That the changes are similar for the three samples is consistent with the overall similarity of the stress patterns (Fig. 7), but the differences between the samples are smaller than in any other rheological characteristic. This shows the difference in rheological response between changing the degree of dispersion and changing the strength of the shear flow.

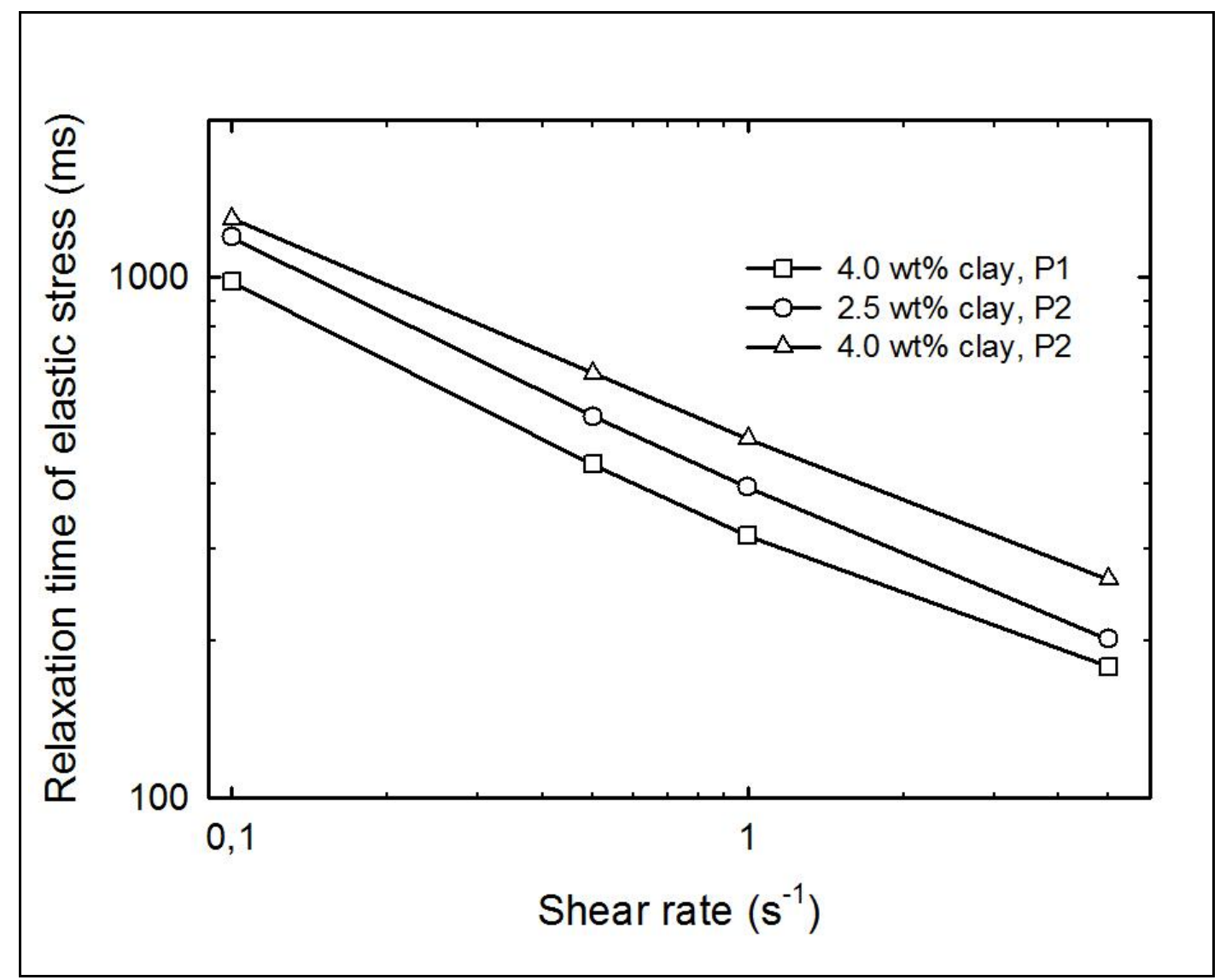

Fig. 8 Relaxation times of the elastic stress contribution, effect of clay concentration, preparation procedure and shear rate 


\section{Flow reversal and intermittent flow}

For structured systems, flow reversal (FR) has proved to be an effective technique to probe the microstructure, in particular its anisotropy (Moldenaers et al. 1991). In intermittent flows, the flow can be started up again after a rest period in either the forward (FF) or the reverse (FR) direction of the previous flow. Solomon and coworkers (2001) observed a stress overshoot in FR experiments on clay/polymer nanocomposites and found that its magnitude was dependent on the amount of rest time allowed between preshearing and the flow reversal measurement. This is the typical response of thixotropic systems (Mewis and Wagner 2009). Letwimolnun and coworkers (2007) employed FR measurements to investigate the effect of the state of dispersion and found that the magnitude of the overshoots increased with the degree of clay exfoliation in the matrix. By comparing start-up flows after various rest periods in the direction of the previous shearing with those in the reverse one, the relaxation of flow-induced anisotropy can be followed. The results for clay/polymer nanocomposites indicate that anisotropy decays very slowly in these materials (Letwimolnun et al. 2007; Vermant et al. 2007; Sung et al. 2008). Mobuchon and coworkers (2007) observed that the stress transients in flow reversal displayed two overshoots. They attributed the first overshoot to the breakdown of structure that had built up during rest, the second overshoot was associated with the breakdown of structure as rearranged during the interval time between the first and second overshoot. 


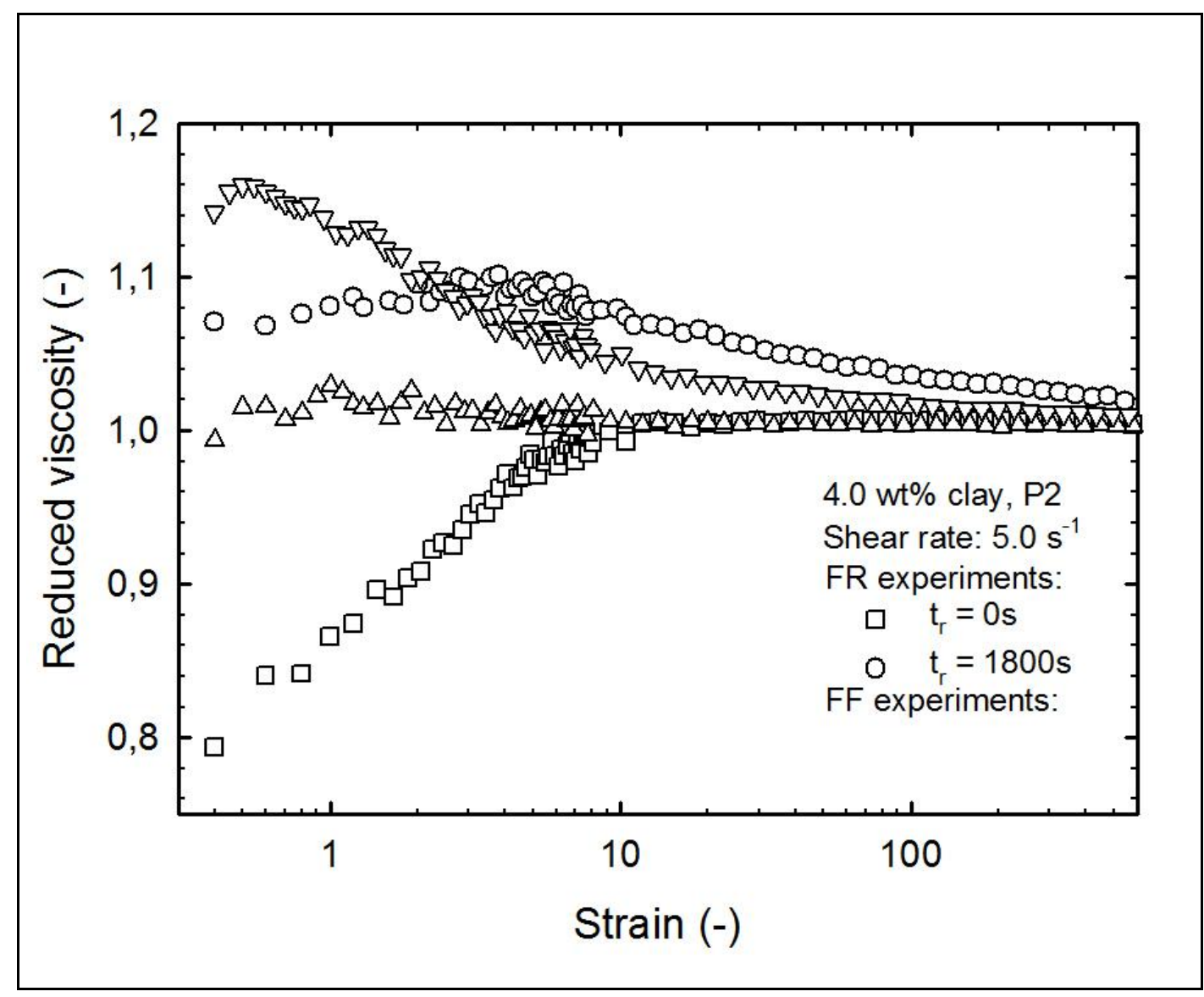

Fig. 9 Transient viscosities, scaled by their steady state values, during start-up flow in the forward (FF) and reverse (FR) directions at a shear rate of $5.0 \mathrm{~s}^{-1}$, effect of waiting time (4.0 wt\% clay, preparation procedure $\mathrm{P} 2$ )

In Fig. 9 stress transients during start-up flow in the forward and reverse directions are compared after shearing at $5 \mathrm{~s}^{-1}$ for the $4 \mathrm{wt} \% \mathrm{P} 2$ sample. The reduced stresses are scaled with their steady state values. In start-up in the forward direction after a rest period of $1 \mathrm{~s}$, the stresses regain their steady state value quasi immediately. This is not the case in the instantaneous flow reversals, where the stress rapidly jumps to about $80 \%$ of the steady state value, after which it takes several strain units to reach the final value. The difference between the two types of start-up flow indicates the presence of an anisotropic structure, a common result for clay/polymer systems. The transients in reverse flow reflect a rearrangement or reorientation of the structural elements. When starting up after a period of rest, the stresses initially reach higher values in both types of experiments, as illustrated in Fig. 9 for a rest period of 1800 s. Yet, additional changes over the next few strain units remain visible in flow reversal. The curves are reminiscent of those with two maxima reported earlier (Mobuchon et al. 2007). The stress growth at small strains indicates that additional links develop between the particles or flocs, the differences in the subsequent evolution at larger strain suggests that the original anisotropic structure maintains its identity during this growth. This latter result is consistent with rheo-optical 
measurements on clay/nanocomposites (Mobuchon et al. 2009). The increase of the peak stress with time in the forward flow experiments seems therefore a more direct indication of the strength of the structure at rest. This was not the case for the data of Letwimolnum et al. (2007), where reverse flow turned out to be more suitable. The effect of structure growth on the peak stress is at any rate much smaller than the increase of the dynamic moduli at rest.

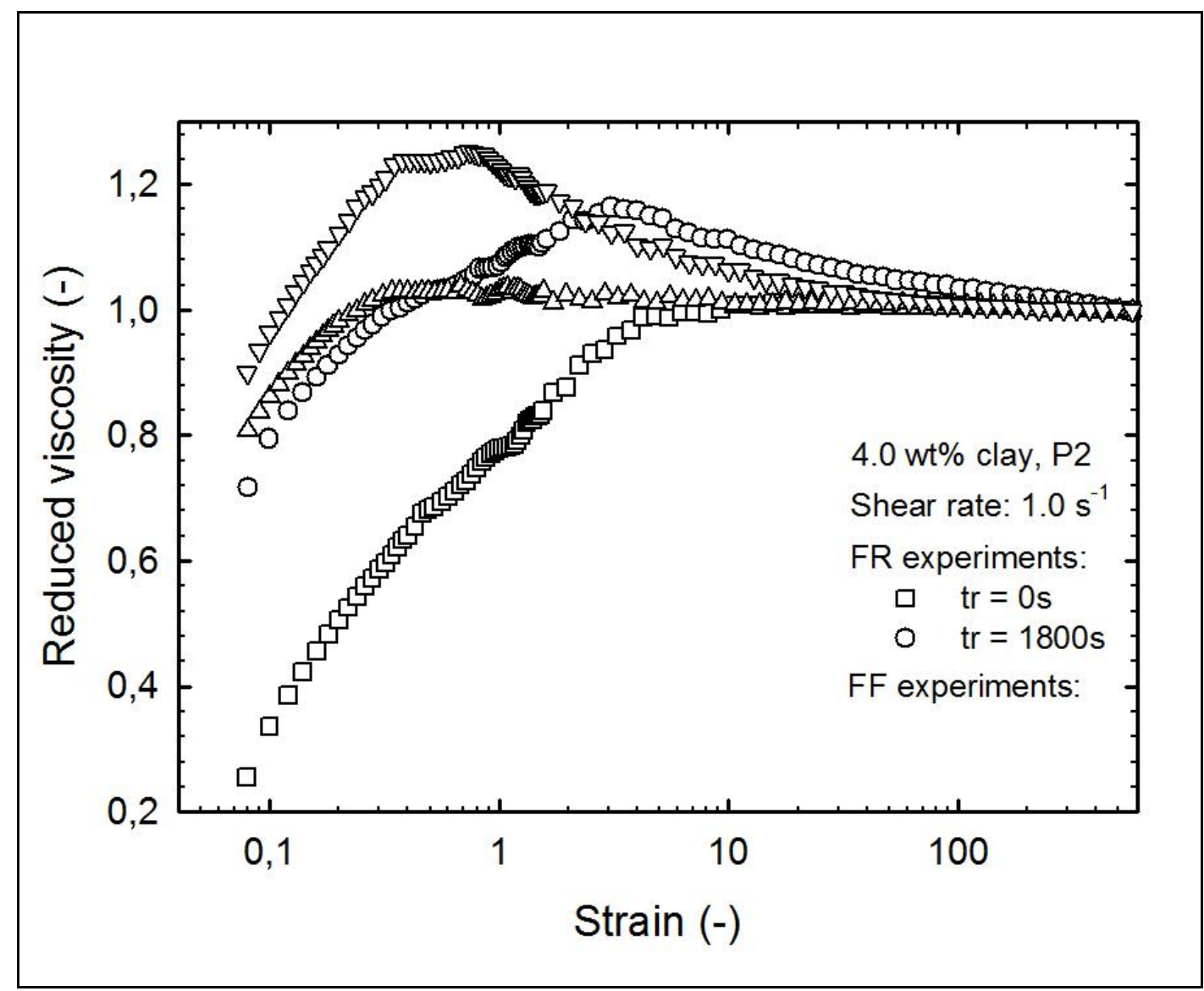

Fig. 10 Transient viscosities, rescaled by their steady state values, during start-up flow in forward and reverse directions at a shear rate of $1.0 \mathrm{~s}^{-1}$, effect of waiting time (4.0 wt\% clay, preparation procedure $\mathrm{P} 2$ )

The start-up curves in forward and reverse flow after different rest periods depend on shear rate. The data in Fig. 9 for $5 \mathrm{~s}^{-1}$ can be compared with those in Fig. 10 for $1 \mathrm{~s}^{-1}$. The structure during flow is now more developed, which results in a larger elastic stress component, as discussed in the previous section. Here, this results in a more gradual stress growth at $1 \mathrm{~s}^{-1}$, even for the FF start-up curve. The stress growth is also more visible at the lower shear rate because data can be taken already at lower strains. The existence of an anisotropic structure is obvious at this shear rate. Again the stresses in flow reversal peak only after several strain units. Similarly, the more gradual rise of the stresses in flow reversal is again preserved after extended rest periods. At still lower shear 
rates than $1 \mathrm{~s}^{-1}$ (not shown), the stresses in the reverse transients evolve even more gradual. The various results for the $4 \mathrm{wt} \% \mathrm{P} 1$ sample were very similar to those shown here for the $4 \mathrm{wt} \% \mathrm{P} 2$ sample. The difference in structure between the two preparation methods did hardly show up in the transients and did not create a substantial difference in anisotropy.

\section{Effect of shear}

Except for the preparation procedure, shear rate and shear history are the main factors that dominate the evolution of microstructure in clay/polymer nanocomposites. This was already obvious from the stress jump and intermittent flow measurements presented above. A striking example is also provided by the effect of the previous shear rate on the subsequent recovery of the moduli. In Fig. 11 the effect of shear history on structure development is shown for a sample with $3 \mathrm{wt} \%$ clay, prepared according to procedure P2. The sample is first sheared at $20 \mathrm{~s}^{-1}$ during two hours. This breaks down the reversible microstructure more or less completely. Subsequently, the shear flow is either arrested or reduced to 0.01 or $0.03 \mathrm{~s}^{-1}$. In each case the structure build-up is followed by intermittently measuring the storage moduli at a fixed frequency of $0.325 \mathrm{rad} / \mathrm{s}$. In Fig. 11 the results are compared with those with previous shearing at $5 \mathrm{~s}^{-1}$.

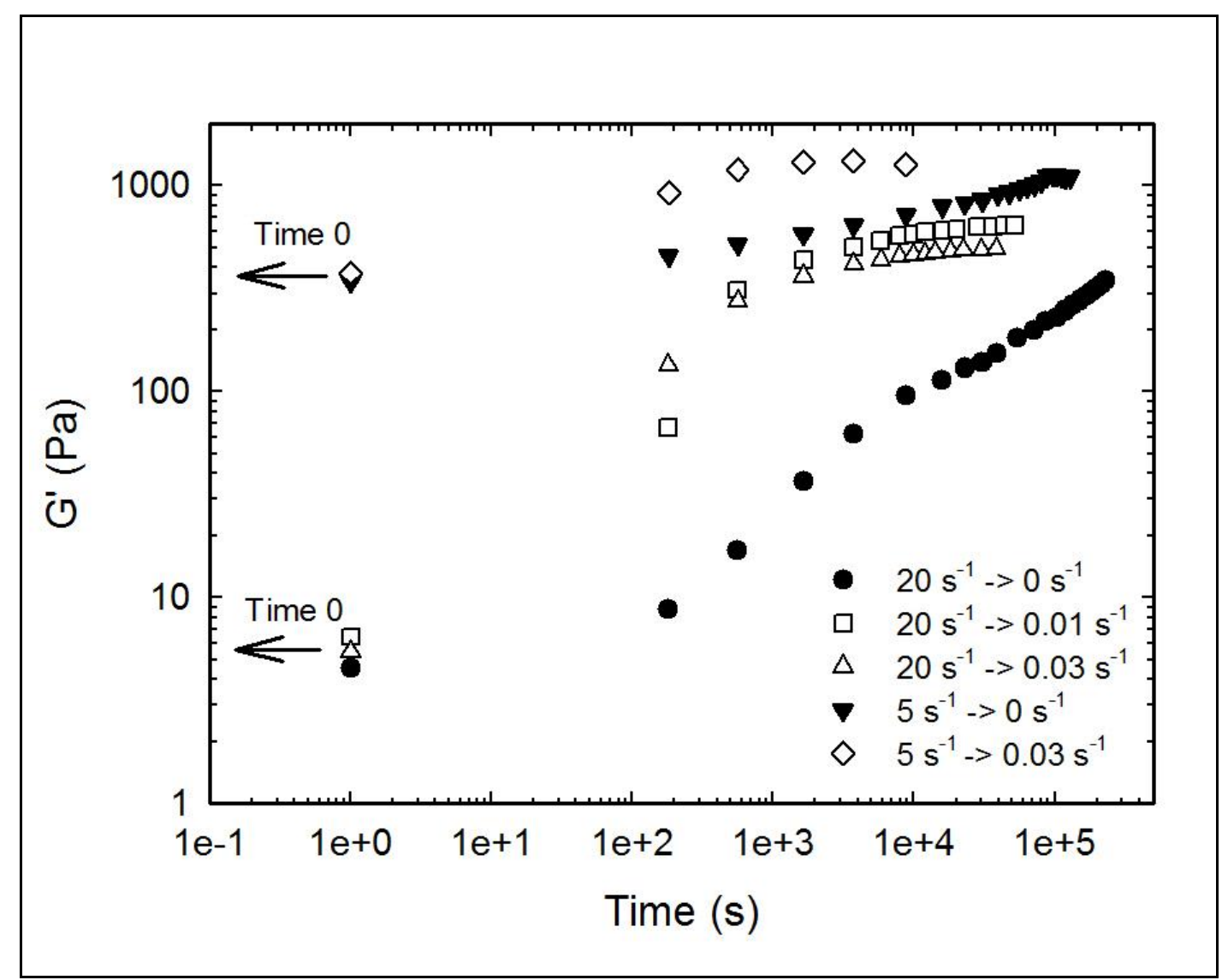


Fig. 11 Recovery at rest and during shear after shearing at 20 and $5 \mathrm{~s}^{-1}$ measured at $\omega=$ $0.325 \mathrm{rad} / \mathrm{s}$ ( $3 \mathrm{wt} \%$ clay, preparation procedure $\mathrm{P} 2$ )

After shearing at $20 \mathrm{~s}^{-1}$ the moduli at rest increase faster than after shearing at $5 \mathrm{~s}^{-1}$ but full recovery clearly is still a slow process. Applying a small shear rate during recovery changes the picture drastically: moduli that at rest are only reached after $10^{5} \mathrm{~s}$ are obtained under shear after $500 \mathrm{~s}$. A tenfold rise of the moduli occurs within a few hundred seconds at a shear rate of $0.01 \mathrm{~s}^{-1}$, i.e. during a total deformation of just a few strain units. The maximum level that the moduli reach seems to decrease with increasing applied shear rate, consistent with earlier results. Structure build-up is also expected to depend on the initial conditions, i.e. the shear rate at which the sample is preconditioned. After shearing at $5 \mathrm{~s}^{-1}$ the initial moduli are much higher because of the lower initial shear rate, but the relative increase in time is smaller when compared at the same recovery conditions. Still, the long term values reached by the moduli are higher, as was reported earlier (Mobuchon et al. 2007). It thus must be concluded that shearing has a significant effect on structure recovery. Applying low shear rates during structure build-up, clearly accelerates the recovery of the moduli. It should be pointed out however that when the same level of moduli is reached at rest as during shear, one does not necessarily have the same structure.

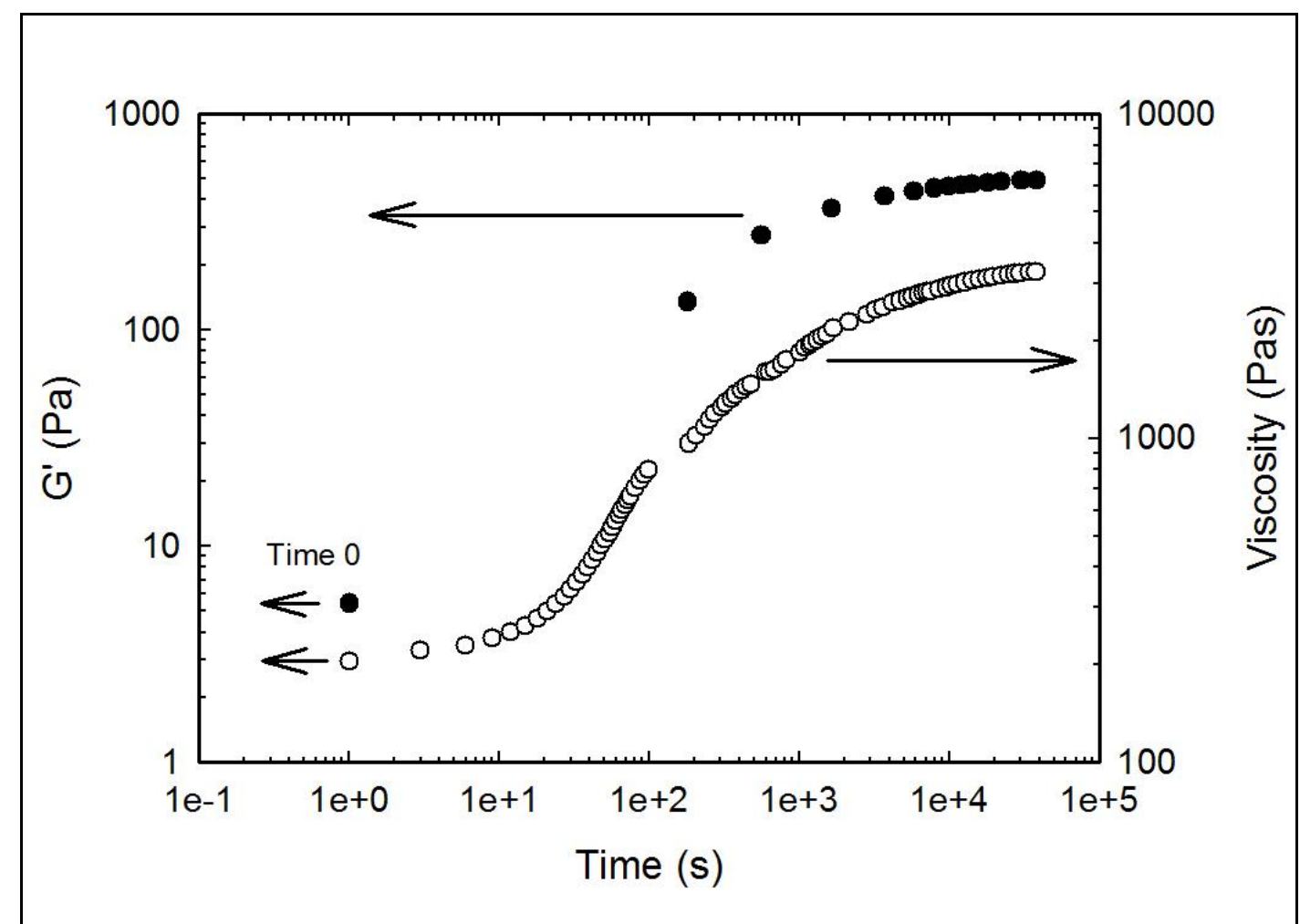

Fig. 12 Comparison of structure recovery after reducing the shear rate from 20 to $0.03 \mathrm{~s}^{-1}$, measured either by the viscosity or the storage modulus at $0.325 \mathrm{rad} / \mathrm{s}$ 
As an alternative method to the dynamic moduli used in Fig. 11, the thixotropic structure recovery after a sudden decrease in shear rate can be followed by measuring the transient viscosity. The two techniques are compared in Fig. 12 for a sudden drop in shear rate from 20 to $0.03 \mathrm{~s}^{-1}$. The measurements of moduli and viscosity have been performed during the same run. For that purpose the steady state shear flow was briefly interrupted at various times to allow for a dynamic measurement. This was possible because of the slow recovery at rest during the dynamic measurements. The curves evolve roughly over the same time range. The low frequency moduli change over two orders of magnitude whereas the steady state viscosity only increases by one order of magnitude. As pointed out in the previous paragraph, the moduli in this experiment do not measure the same structure evolution as in a recovery experiment at rest. During shear, as in the present experiment, the moduli follow more closely the development of gradually growing flocs during shear. Network formation is made impossible by the continuous flow. Instead, the storage moduli probe the elasticity of the flocs comparable with the elastic stresses in stress jump experiments. When, on the other hand, the flow is stopped, the flocs that exist at that moment do not grow gradually anymore but instead aggregate in larger entities that readily form a network as illustrated by a plateau for $\mathrm{G}^{\prime}$ at low frequencies. Hence, by altering the shear history, a large variety of different structures can be generated.

\section{Conclusions}

The dispersion procedure affects the level and kinetics of the dynamic moduli after cessation of flow. In particular the time to reach the crossover point can be highly sensitive to differences in microstructure. The moduli-frequency curves for different degrees of dispersion, different clay contents and different rest time can be superimposed by the scaling procedure for attractive colloids. Deviations occur for the low frequency loss moduli. These suggest the presence of a relaxation mechanism with a very large relaxation time that does not follow the same scaling. Plotting the modulus at crossover versus the crossover frequency for different recovery times results in parallel lines for the different concentrations. The lines for the different dispersion procedures coincide. The slope of the line does not fit the one required by the scaling law.

With stress jump experiments, the hydrodynamic and elastic components of the steady state stresses have been determined. The stresses during shear are logically less affected by differences in microstructure than the low frequency moduli at rest. Significantly, the relaxation times of the elastic stress components are hardly sensitive to differences in dispersion procedure or clay content, but they change drastically when the structure is 
altered by means of the shear rate. The effect of shear rate prior to structure recovery as well as during the recovery was investigated. Applying even a low shear rate during recovery caused a substantial increase in recovery rate, even at total strains of only a few strain units. The evolution of anisotropy during structure recovery after cessation of flow is followed with startup experiments in the flow and the reverse directions. A structure rearrangement over several strain units only in reverse flow demonstrates the anisotropy. This rearrangement remains visible even after long rest periods, indicating that the anisotropic element in the microstructure preserves its identity during recovery. The results are less sensitive to the degree of dispersion. The final conclusion is that different rheological measurements probe different aspects of the microstructure, including different sensitivity to changes induced by the degree of dispersion or by shear.

\section{Acknowledgements}

Support of the Onderzoeksfonds K.U.Leuven under grant GOA03/06 and GOA09/002 is gratefully acknowledged. R. Cardinaels is indebted to the Research Foundation - Flanders (FWO) for a doctoral scholarship. The clay sample and the XRD measurements have been generously provided by Dr. W. IJdo (Elementis International). The authors thank Prof. B. Goderis for performing the SAXS measurements.

\section{References}

Aral BK, Kalyon DM (1997) Viscoelastic material functions of noncolloidal suspensions with spherical particles. J Rheol 41:599-620

Batchelor GK (1970) The stress system in a suspension of force-free particles. J Fluid Mech 41:545-570

Barnes HA (1997) Thixotropy- A review. J Non-Newtonian Fluid Mech 70:1-33

Bousmina M (2006) Study of intercalation and exfoliation processes in polymer nanocomposites. Macromolecules 39:4259-4263. DOI: 10.1021/ma052647f

Carreau PJ, Lavoie PA, Bagassi M (1996) Rheological properties of filled polymers. Macromol Symp 108:111-126

Dullaert K, Mewis J (2005) Stress jumps on weakly flocculated dispersions: steady state and transient results. J Colloid Interface Sci 287:542-551. DOI: 10.1016/j.jcis.2005.02.018 
Gilman JW (1999) Flammability and thermal stability studies of polymer layered-silicate (clay) nanocomposites. Appl Clay Sci 15:31-49

Gilman JW, Jackson CL, Morgan AB, Harris R, Manias E, Giannelis EP, Wuthenow W, Hilton D, Phillips SH (2000) Flammability properties of polymer-layered-silicate nanocomposites. polypropylene and polystyrene nanocomposites. Chem Mater 12:18661873

Kobelev V, Schweizer KS (2005) Nonlinear elasticity and yielding of depletion gels. J Chem Phys 123, 164902. DOI: 10.1063/1.2109887

Krishnamoorti R, Yurekli K (2001) Rheology of polymer layered silicate nanocomposites. Curr Opin Colloid Interface Sci 6:464-470

Le Meins JF, Moldenaers P, Mewis J (2002) Suspensions in polymer melts. 1. Effect of particle size on the shear flow behavior. Ind Eng Chem Res :6297-6304. DOI: 10.1021/ie020117r

Letwimolnun W, Vergnes B, Ausias G, Carreau PJ (2007) Stress overshoots of organoclay nanocomposites in transient shear flow. J Non-Newtonian Fluid Mech 141:167-179. DOI: 10.1016/j.jnnfm.2006.11.003

Liu L, Qi Z, Zhu X (1999) Studies on nylon 6/clay nanocomposites by melt-intercalation process. J Appl Polym Sci 71:1133-1138

Mackay ME, Liang CH, Halley PJ (1992) Instrument effects on stress jump measurements. Rheol Acta 31:481-489

Mackay ME, Kaffashi B (1995) Stress jumps of charged colloidal suspensions, measurement of the elastic-like and viscous-like stress components. J Colloid Interface Sci 174:117-123

Messersmith PB, Giannelis EP (1995) Synthesis and barrier properties of poly( $\varepsilon^{-}$ caprolactone)-layered silicate nanocomposites. J Polym Sci Part A: Polym Chem 33:1047-1057

Mewis, J, Wagner NJ (2009) Thixotropy. Adv Colloid Interf Sci 147-148:214-227. DOI: 10.1016/j.cis.2008.09.005

Mobuchon C, Carreau PJ, Heuzey M-C (2007) Effect of flow history on the structure of a non-polar polymer/clay nanocomposite model system. Rheol Acta 46:1045-1056 DOI: $10.1007 / \mathrm{s} 00397-007-0188-5$

Mobuchon C, Carreau PJ, Heuzey M-C, Reddy NK, Vermant J (2009) Anisotropy of nonaqueous layered silicate suspensions subjected to shear flow. J Rheol 53: 517-538. DOI: 10.1122/1.3094911 
Moldenaers P, Yanase H, Mewis J (1991) Flow-induced anisotropy and its decay in polymeric liquid crystals. J Rheol 35: 1681-1699.

Morgan AB, Gilman, JW (2003) Characterisation of polymer-layered silicate (clay) nanocomposites by transmission electron microscopy and X-ray diffraction: A comparative study. J Appl Polym Sci 87:1329-1338. DOI: 10.1002/app.11884

Nazockdast E, Nazockdast H, Goharpey F (2008) Linear and nonlinear melt-state viscoelastic properties of polypropylene/organoclay nanocomposites. Polym Eng Sci 48:1240-1249. DOI: 10.1002/pen.21054

Pignon F, Magnin A, Piau J-M (1998) Thixotropic behavior of clay dispersions: Combinations of scattering and rheometric techniques. J Rheol 42:1349-1373

Prasad V, Trappe V, Dinsmore AD, Segre PN, Cipelletti L, Weitz DA (2003) Universal features of the fluid to solid transition for attractive colloidal particles. Faraday Discuss 123:1-12. DOI: 10.1039/b211107c

Ren JX, Krishnamoorti R (2003) Nonlinear viscoelastic properties of layered-silicatebased intercalated nanocomposites. Macromolecules 36:4443-4451. DOI: $10.1021 / \mathrm{ma} 020412 \mathrm{n}$

Romeo G, Filippone G, Fernandez-Nieves A, Russo P, Acierno D (2008) Elasticity and dynamics of particle gels in non-Newtonian melts. Rheol Acta 47:989-997. DOI: 10.1007/s00397-008-0291-2

Solomon MJ, Almusallam AS, Seefeldt KF, Somwangthanaroj A, Varadan P (2001) Rheology of polypropylene/clay hybrid materials. Macromolecules 34:1864-1872

Sung JH, Mewis J, Moldenaers P (2008) Transient rheological probing of PIB/hectoritenanocomposites. Korean-Austr. Rheol J 20:27-34

Trappe V, Weitz DA (2000) Scaling of the viscoelasticity of weakly attractive particles. Phys Rev Lett 85:449-452

Vaia RA, Price G, Ruth PN, Nguyen HT, Lichtenhan J (1999) Polymer/layered silicate nanocomposites as high performance ablative materials. Appl Clay Sci 15:67-92

Vermant J, Ceccia S, Dolgovskij MK, Maffettone PL, Macosko CW (2007) Quantifying dispersion of layered nanocomposites via melt rheology. J Rheol 51:429-450. DOI: $10.1122 / 1.2516399$

Wang Z, Pinnavaia TJ (1998) Nanolayer reinforcement of elastomeric polyurethane. Chem Mater 10:3769-3771 
Winter, HH, Chambon F (1986) Analysis of linear viscoelasticity of a crosslinking polymer at the gel point. J Rheol 30:367-382

Yano K, Usuki A, Okada A (1997) Synthesis and properties of polyimide-clay hybrid films. J Polym Sci Part A: Polym Chem 35:2289-2294

Zeng C, Lee LJ (2001) Miscibility and hydrogen bonding in blends of poly(vinylphenolco-methyl methacrylate) with poly(ethylene oxide). Macromolecules 34:4098-4103

Zhao, J, Morgan AB, Harris JD (2005) Rheological characterization of polystyrene-clay nanocomposites to compare the degree of exfoliation and dispersion. Polymer 46:86418660. DOI: $10.1016 /$ j.polymer.2005.04.038 


\section{Captions for figures}

Fig. 1 SAXS patterns of pure clay and of two polymer/clay nanocomposites obtained with a different dispersion procedure

Fig. 2 Recovery of the dynamic moduli after shearing at $5 \mathrm{~s}^{-1}$ for 10 minutes, strain amplitude $=0.01$; (a) $4.0 \mathrm{wt} \%$ clay, preparation procedure $\mathrm{P} 1$; (b) $4.0 \mathrm{wt} \%$ clay, preparation procedure $\mathrm{P} 2$

Fig. 3 Scaled modulus-frequency curves after various rest times (data of Fig. 2 and of a $2.5 \mathrm{wt} \%$ sample prepared according to procedure P2)

Fig. 4 Crossover moduli $G_{c}$ as a function of crossover frequency $\omega_{c}$ for suspensions with different clay contents and varying waiting times

Fig. 5 Evolution of the dynamic moduli at $1 \mathrm{rad} / \mathrm{s}$ after shearing for 10 minutes at $5 \mathrm{~s}^{-1}$, effect of particle concentration and preparation procedure

Fig. 6 Stress jump measurements: initial stress relaxation after shearing at $0.1,0.5,1.0$ and $5.0 \mathrm{~s}^{-1}$ for the sample with $2.5 \mathrm{wt} \%$ clay (preparation procedure $\mathrm{P} 2$ )

Fig. 7 Effect of preparation procedure on the elastic ( $\square$ ) and hydrodynamic (o) contributions to the total steady state stress $(\Delta)$; (a) $4.0 \mathrm{wt} \%$ clay, preparation procedure $\mathrm{P} 1$; (b) $4.0 \mathrm{wt} \%$ clay, preparation procedure $\mathrm{P} 2$

Fig. 8 Relaxation times of the elastic stress contribution, effect of clay concentration, preparation procedure and shear rate

Fig. 9 Transient viscosities, scaled by their steady state values, during start-up flow in the forward (FF) and reverse (FR) directions at a shear rate of $5.0 \mathrm{~s}^{-1}$, effect of waiting time (4.0 wt\% clay, preparation procedure $\mathrm{P} 2$ )

Fig. 10 Transient viscosities, rescaled by their steady state values, during start-up flow in forward and reverse directions at a shear rate of $1.0 \mathrm{~s}^{-1}$, effect of waiting time (4.0 wt $\%$ clay, preparation procedure $\mathrm{P} 2$ )

Fig. 11 Recovery at rest and during shear after shearing at 20 and $5 \mathrm{~s}^{-1}$ measured at $\omega=$ $0.325 \mathrm{rad} / \mathrm{s}$ ( $3 \mathrm{wt} \%$ clay, preparation procedure $\mathrm{P} 2$ )

Fig. 12 Comparison of structure recovery after reducing the shear rate from 20 to $0.03 \mathrm{~s}^{-1}$, measured either by the viscosity or the storage modulus at $0.325 \mathrm{rad} / \mathrm{s}$ 